\section{CONSOLIDATION OF ANNUAL CMD GENERAL CONFERENCE}

Two years ago we reported in Europhysics News (May, 1980) on, what was called "new beginnings", the first General Conference of the Condensed Matter Division of the European Physical Society in Antwerp which had attracted 650 solid state scientists. Considerable enthusiasm was expressed in Antwerp for both the programme (with 9 plenary talks, 65 invited speakers and 320 contributed papers) and the prospect of establishing a series of conferences on the same lines. Immediately after the Conference, a charter was established by the CMD Board which defined the future General CMD Conferences as independant European ventures, and it was also decided that the 1982 meeting would take place in Manchester (with Prof. Heine as Conference Chairman) and the 1983 meeting in Lausanne (with Prof. E. Mooser as Chairman). Since then the Hague has been designated for 1984 (Prof. F. Mueller) and discussions have been opened with the Fed. Rep. of Germany for 1985 (Prof. J. Treusch).

The challenge for Prof. Heine and his colleagues was not minor as it still had to be proved that a programme could be devised which would attract many solid state physicists on an essentially annual basis. Great care was taken to ensure a wide consultation in the choice of speakers. A large international Advisory Committee was formed to help the International Programme Committee and the names of suggested speakers were recirculated before final selection. When the tentative programme then became known many contributed papers were introduced, some of such calibre that additional invited talks were added.

The atmosphere of the Manchester meeting was one of strong scientific interaction (not the least at the poster sessions) and many new scientific contacts were made. I was especially impressed by the positive response of the younger participants and their ready, almost automatic, acceptance of the European approach.

This identification with the wider scientific community was particularly evident in the open meeting of the CMD and is reflected in the Board's decision to involve Individual Members of EPS in the selection of invited speakers for 1984 . The Board also decided for future CMD General Conferences to publish invited papers (but not necessarily contributed papers). Restricted proceedings will after all be produced for Manchester although no pressure will be put on the invited speakers to write up their contributions.

J.T. Devreese, Chairman of the EPS Condensed Matter Division

\title{
2nd General Conference of the CMD
}

\author{
Some personal impressions by Volker Heine, Chairman of the \\ Conference, J.T. Devreese, Chairman of the EPS Condensed \\ Matter Division and F.M. Mueller of Nijmegen.
}

Condensed Matter ('Solid State') Physics covers $40 \%$ of all physics, as judged by the number of column-centimetres in the Physics Abstracts Classification Scheme. Magnetism, semiconductor devices, surface physics, etc. are in one sense quite separate disciplines with their own phenomena, principles and experimental techniques. Yet there are also diverse threads running through this cluster of subjects. Superionic conductors and polymers have related transport equations, 'spin-glasses' occupy a niche between distant territories....... and so on.

These interconnections provide the raison d'être of the Annual General Conferences of the Condensed Matter Division, the second of which was held over $3 \frac{1}{2}$ days in warm sunshine in Manchester, UK on 22-25 March 1982. Its success with 600 participants sets the seal on the new annual series. A solid state physicist cannot afford to ignore cross-fertilisation from neighbouring fields to his own, but apart from that, a lot of the fun in solid state physics stems from the vast diversity of phenomena and materials. The scope and vitality of both the field and the EPS Condensed Matter Division is indicated by the fact that over 20 specialised conferences, workshops and summer schools were organised in 1981 under the auspices of the Division's various Sections. With a larger number of topical conferences, Europe has probably a better balance between general and specialised conferences than the USA, where the March Meeting has become so huge, because it does double duty as general conference and specialised conferenceswithin-the-conference.

Let diversity therefore be the theme around which to mention some of the highlights of the 57 invited talks and 360 contributed papers. Of course, no one could attend them all, necessarily placed in parallel sessions as most were; but then cross-fertilization does not come with equal probability from all directions, so that people like to select to some degree. The most striking diversity is the range from industrial applications to the most fundamental principles of quantum mechanics. A.J. Leggett in "Quantum Noise and Quantum Tunnelling in Macroscopic Systems" asked whether the present formulation of quantum mechanics is valid for macroscopic systems, in particular as regards the Schrödinger cat paradox about what constitutes a measurement. Prelimi- nary experimental results on tunnelling of flux quanta in SQUID rings deviate from theoretical expectations but a more detailed analysis of fluctuations is required. P.W. Anderson in "Overview of the Localisation Problem" also discussed how recent measurements in disordered materials including two-dimensional systems required a deep analysis of macroscopic concepts such as electrical resistance in fundamental quantum terms. When interatomic spacing, wave function coherence length and mean free path for inelastic scattering become comparable, saturation and logarithmic dependence on parameters can result. Tying different dimensions and ranges of parameters together are the scaling laws based on the principle that one resistance specimen can always be regarded as the sum of two smaller ones.

At the opposite extreme the symposium on "Physics of Optical Transmission Systems" touched on the frontiers of modern technology. K.H. Zschauer from Siemens discussed the semiconductor lasers and receivers at the ends of the optical fibre, whereas other speakers focussed on the physics of the fibre itself, and on methods for making purely optical switches, amplifiers etc. In each case optimising the design for technological purposes requires a deeper understanding and hence stimulates new good physics. Other talks with partly an applied physics orientation included "Materials Research for Fusion Energy" by J. Nihoul, "Polymer/Dopant Systems with Electronic Conductivity" by P. Bernier, and two on amorphous metals by K.H.J. Buschow and H.J. Güntherodt. Unfortunately there were not many contributed papers from physicists in industry. For them, the important cross-fertilisation is between device engineering and the basic science of semiconductors or magnetism etc. as the case may be, which they find at the relevant topical international conferences. But the conference chairman, in his concluding remarks, warned the basic physics community against cutting itself off from the technological applications which, as already noted, stimulate new physics, generate jobs for our graduates, and ultimately provide the reason why governments pour large sums of money into scientific research.

A second diversity axis stretches from progress on old questions to brand new developments. For example, N. Wiser reviewed how recent work had cleared up 
long-standing puzzles about the temperature dependence of the electrical resistivity and related transport coefficients of the alkali and other simple metals. At the other end, to highlight the most recent interesting advances in research, the conference organisers picked out 11 of the contributed abstracts and had them presented as special 20 minute talks under the heading "Selected Contributed Papers". In one of these, G. Binnig and H. Rohrer described a new tool, the scanning tunnelling microscope which can easily resolve single atomic steps on a surface. Strong evidence for soliton transport in the quasi one-dimensional salt TMMC was presented by J.A.H.M Buys and W.J.M. de Jonge, while R. Cotterill and J.U. Madsen showed, by molecular dynamics computations, that the entropies of the glassy state and of the crystal are the same at low temperature for spheres with a Lennard-Jones potential. Aside from those specially selected and the talks of a few participants who specifically requested oral presentation, all other contributed papers were given in six wellarranged poster sessions. A lively buzz surrounded the posters and most participants seemed to report good interactions.

A few invited papers were specifically chosen to keep solid state physicists in touch with other research on their boundaries. A symposium by P. Collet, R. Berge and M. Giglio described beautiful theory and experiments on "The Transition to Chaotic Behaviour" i.e. to turbulence. Sir Sam Edwards discussed ordinary and unusual phase transitions in polymers illustrating it with a movie film of a fluidised column of 4 inch nails, while S. Doniach took us to the application of synchrotron radiation as a tool in chemistry and biology.

Several American and Canadian physicists - and others from even further afield - were welcomed at the meeting, some attracted by the $1 \frac{1 / 2}{2}$ day satellite conferences on charge density waves and on ultra-low temperature physics, or the workshop on deep traps. Eighty people went on a half-day visit to the new dedicated synchrotron at Daresbury. Incidentally, it was good to see that women participants had advanced in numbers from a conspicuous rarity to a tiny minority, about $4 \%$ as a subjective estimate. They included one invited speaker S. Popova from the USSR and two presenters of the specially Selected Contributed Papers. M. Combescot described work with $\mathrm{J}$. Bok on a high temperature instability of the electron-hole system in silicon with its possible role in melting, while A. Bussmann-Holder spoke about a theory developed with $\mathrm{H}$. Buttner and $\mathrm{H}$. Bilz to explain the commensurate-incommensurate transition in $\mathrm{K}_{2} \mathrm{SeO}_{4}$ with the puzzling wave vector near to one-third of a reciprocal lattice vector.

Altogether there was a sparkle of exciting new physics about the whole confe- rence, in both the invited and contributed papers. It is tempting for solid state physicists to feel that the cream has been licked from their pot: after all, magnetic alloys, superconductivity etc. have been around for a long time, and indeed there is much important bread-and butter work being done. But the conference displayed there is also a continually renewing challenge in the field, coming from new materials, technological challenges, advances in experimental techniques, and phenomena that may not be new but had not really been in the main-stream of scientific interest, sometimes because they had been too difficult to come to grips with before. These developments make new demands on understanding: it is not a matter of "just another band structure calculation".

\section{V. $H$.}

\section{Additional Reflections on the Physics}

M.L. Cohen gave an overview of the considerable progress which has been made over the past years concerning $a b$ initio calculations of the total energy of semiconductors. Although, of course, approximations are involved concerning the many-body aspects of the problem (even using non-local pseudopotentials one does not solve the exchange-correlation problem rigorously) results of amazing accuracy are obtained for fundamental quantities like the lattice parameter (at the $1 \%$ level and better e.g. for $\mathrm{Si}$ or $\mathrm{GaAs}$ ) while the only input to the computer program consists of atomic numbers and a few possible lattice structures. These studies have been extended to include surface properties (e.g. surface states).

Among the potential applications of the total energy correlations are also "frozen" phonon calculations which allow evaluation of phonon frequencies especially at high symmetry point of the Brillouin zone. Two computational methods to derive phonon spectra were mentioned by Cohen:

i) derivation via total energy calculations (the total energy is calculated for the groundstate of the crystal and for different high symmetry "frozen phonon states". This method, pioneered also by Richard Martin, was applied to Si by Cohen (1981) and co-workers and to GaAs by Martin and Kunc (1980). The method also allows for the study of Grüneisen parameters:

ii) Cohen also mentioned the dielectric formalism to calculate phonon dispersion curves in semiconductors. This technique which gives no information on the Grüneisen parameter but leads to phonons at all wavelengths, led to adequate phonon dispersion curves in 1979. It was reviewed by $P$. Van Camp the same morning.
Considering only the contributed and poster papers, I would like to add first a few more details to the notes given on the contribution by $\mathrm{G}$. Binnig and $\mathrm{H}$. Rohrer on "Scanning Tunnelling Microscopy: a New Tool". They discussed the feasibility of controlled vacuum tunnelling from a tungsten tip to a Pt surface held $10 \AA$ from each other. The tunnelling distance may be stabilized to within $0.2 \AA$. The importance is the use of this new vacuum tunnelling technique to resolve surface topography, and surface characterization. There seems little doubt that this technique will lead to many novel results in surface studies, and surface kinetics.

J.J.M. Franse and a mostly Dutch group in their paper on the "Suppression of Spin Fluctuations in $\mathrm{UAl}_{2}$ in High Magnetic Fields" dealt with magnetization, magnetoresistivity and differential susceptibility measurements on $\mathrm{UAl}_{2}$ in fields of 35,25 and $8 \mathrm{~T}$ which showed a suppression of spin fluctuations in this material. Using the temperature dependence of the magnetic susceptibilities through the Maxwell relations and existing theories, the suppression of the electronic contribution to the specific heat can be calculated, which is in excellent agreement with previous measurements of Trainor et al. These experiments suggest that the field and temperature effects on the paramagnon contributions are closely related. In addition, since the paramagnon contributions to the magnetoresistance and magnetization are frozen out above $15 \mathrm{~T}$, specific heat and DHVA measurements above this field would also show a similar decoupling of the paramagnons from the electrons.

R. Gerhard et al., described a "Nondestructive Determination of Special Charge Distributions in Thin Dielectrics" by laser-induced passive pulses. This is based on the ablation of a surface using a laser to produce controlled pressure pulses, through which special charge variations can be detected provided the duration of the pressure pulse is short compared to the transit time, $t=s / c$ where $s$ is the thickness and $c$ the velociy of sound. Plans to extend the technique to sound velocity measurements and ultrasonic attenuation look promising, as does a similar idea using piezo-electricity rather then laser ablation.

B.V.B. Sarkissian et al., in their paper on 'The Hybrid State of the Magnetic Superconductor' described a novel superconductor which forms at a temperature below that at which the compound becomes magnetic. This is the first known instance of such a transition element material in which the same electrons enter into both properties. Theories of superconductivity and magnetism may have to be revised, as a result. 